Pacific Northwest

National Laboratory

Operated by Battelle for the

U.S. Department of Energy

\title{
Energy Management and Control System: Desired Capabilities and Functionality
}

\author{
D.D. Hatley \\ R.J. Meador \\ S. Katipamula \\ M.R. Brambley \\ LtCol. Carl Wouden, USAF, Ret.
}

April 2005

Prepared for the U.S. Department of Energy

under Contract DE-AC05-76RL01830 


\title{
DISCLAIMER
}

This report was prepared as an account of work sponsored by an agency of the United States Government. Neither the United States Government nor any agency thereof, nor Battelle Memorial Institute, nor any of their employees, makes any warranty, express or implied, or assumes any legal liability or responsibility for the accuracy, completeness, or usefulness of any information, apparatus, product, or process disclosed, or represents that its use would not infringe privately owned rights. Reference herein to any specific commercial product, process, or service by trade name, trademark, manufacturer, or otherwise does not necessarily constitute or imply its endorsement, recommendation, or favoring by the United States Government or any agency thereof, or Battelle Memorial Institute. The views and opinions of authors expressed herein do not necessarily state or reflect those of the United States Government or any agency thereof.

\author{
PACIFIC NORTHWEST NATIONAL LABORATORY \\ operated by \\ BATTELLE \\ for the \\ UNITED STATES DEPARTMENT OF ENERGY \\ under Contract DE-AC05-76RL01830
}

\author{
Printed in the United States of America \\ Available to DOE and DOE contractors from the \\ Office of Scientific and Technical Information, \\ P.O. Box 62, Oak Ridge, TN 37831-0062; \\ ph: (865) 576-8401 \\ fax: (865) 576-5728 \\ email: reports@adonis.osti.gov
}

\footnotetext{
Available to the public from the National Technical Information Service, U.S. Department of Commerce, 5285 Port Royal Rd., Springfield, VA 22161 ph: (800) 553-6847 fax: (703) 605-6900

email: orders@ntis.fedworld.gov

online ordering: http://www.ntis.gov/ordering.htm

This document was printed on recycled paper.
} 


\section{Energy Management and Control System: Desired Capabilities and Functionality}

D.D. Hatley

R.J. Meador

S. Katipamula

M.R. Brambley

LtCol. Carl Wouden, USAF, Ret.

April 2005

Prepared for

HQ Air Mobility Command (AMC/CEO)

Under a Related Services Agreement with the US Department of Energy

Contract DE-AC05-76RLO 1830

Pacific Northwest National Laboratory

Richland, Washington 99352 



\section{Summary}

Energy management and control system (EMCS) technology has evolved over the past three decades from pneumatic and mechanical devices to direct digital controls (DDC) or computerbased controllers and systems. Today's EMCS systems consist of electronic devices with microprocessors and communication capabilities and utilize widespread use of powerful, lowcost microprocessors and standard cabling communication protocols.

This document discusses functions and capabilities of a typical building/facility EMCS. The overall intent is to provide a building operator, manager or engineer with basic background information and recommended functions, capabilities, and good/best practices that will enable the control systems to be fully utilized/optimized, resulting in improved building occupant quality of life and more reliable, energy efficient facilities.

Most basic functions and some intermediate and advanced functions described in this document are generally required to operate buildings/facilities efficiently. A careful review of the sitespecific requirements is recommended before picking the functional requirements of an EMCS. It should be noted that the basic functions described in this document are generally available in most EMCS systems, while the availability of the intermediate and advanced functions vary by manufacturer. 


\section{Nomenclature}

AFDD

BACnet

BAS

BMS

Cascading Resets

Commissioning

Controller

Economizer

Demand Limiting

DDC

DDE
Automated fault detection and diagnostics involves the use of software tools to analyze the behavior of the building, determine if the performance is unsatisfactory (fault detection) and then isolate or localize the fault to facilitate repair (fault diagnosis). The behavior may be observed in the course of an active functional test performed by the tool or in the course of passive monitoring of routine operation.

BACnet is a data communication protocol for building automation and control networks developed by American Society of Heating, Ventilation, and Air Conditioning Engineers ANSI/ASHRAE 135-1995. A data communication protocol is a set of rules governing the exchange of data over a computer network.

Building automation systems, also known as EMCS.

Building management systems, also known as EMCS.

An example of cascading resets is when both the chilled-water supply temperature as well as the supply air temperature are reset in a sequence.

A process to ensure that EMCS is designed, installed, functionally tested, and capable of being operated and maintained according to the owner's operational needs.

Processes data that is input usually from a sensor, performs logical operations based on the device being controlled and causes an output action to be generated.

Air-side economizers use controllable dampers to increase the amount of outside-air intake into the building when the outside air is cooler than the return air and the building requires cooling.

A control function that enables management of peak demand level by shedding predefined loads when the building/facility demand nears a preset maximum.

Direct digital controls, also known as EMCS.

Direct data exchange is a standard Microsoft Windows ${ }^{\circledR}$ message-passing protocol that defines a mechanism for Windows applications to share information with one another. 
Distributed Facility

Management

EMCS

FMS

Gateways

Interoperability

Latent Cooling

LonTalk

LonWorks

MODBUS

OLE

Ratchet Clause

Sampling Interval

Settling Interval

Temperature Reset
Management of building and facilities that are distributed over a wide geographical region. Although each building has its own EMCS systems, the buildings are also managed remotely from a central location through use of gateways and software applications.

Energy management control systems

Facility management systems, also known as EMCS.

Devices that allow for integration of control networks with internet, so that the devices/controllers on the control network can be accessed through the internet

Ability of a device/controller or a product to work with other devices/ controllers or products without special effort on the part of the customer.

Moisture removal through dehumidification

A communication protocol for LonWorks devices to communicate with each other on a LonWorks network.

A networking platform created by Echelon Corporation.

A standard communication protocol developed by the Modicon Corporation. The MODBUS protocol is often used in facility metering applications.

Object linking and embedding is like DDE, a standard Microsoft Windows ${ }^{\circledR}$ message-passing protocol, which defines a mechanism for Windows applications to share information with one another.

A term in a customer's contract or rate schedule (tariff) that dictates that the customer's billings for each billing period must be based at least in part on the maximum billing received by that customer over a given period, usually the preceding year.

The interval between two consecutive adjustments of control parameters.

Time required for the control to settle after a change in a control parameter.

Adjustment of supply temperature higher (during cooling) or lower (during heating) to match the heating and cooling needs.

Web-Enabled Device - Device that can be directly accessed from a standard internet browser. 


\section{Contents}

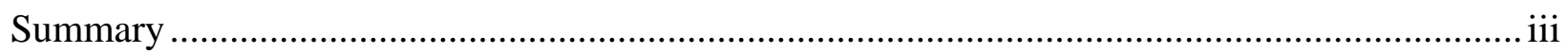

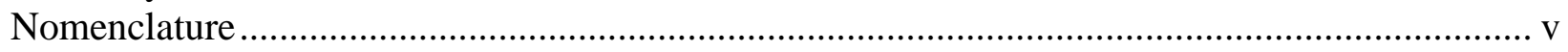

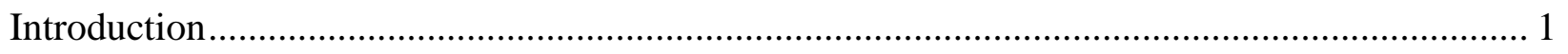

Functions and Capabilities of Energy Management Control Systems (EMCS) ........................... 3

Central Information Processing and Control Workstations ................................................. 4

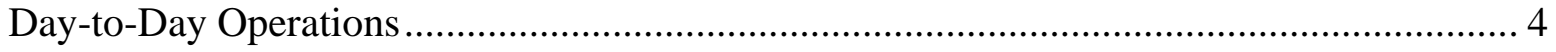

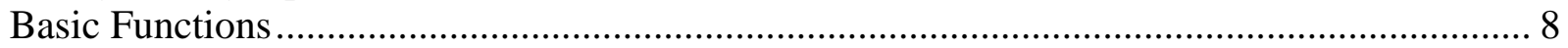

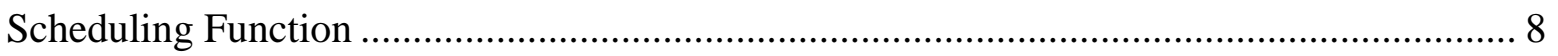

Chilled-Water Reset Function.................................................................................. 9

Hot-Water Reset Function ...................................................................................... 9

Supply-Air Temperature Reset Function ................................................................. 10

Static Pressure Reset Function.......................................................................... 10

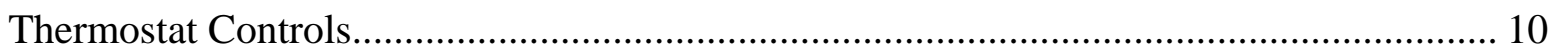

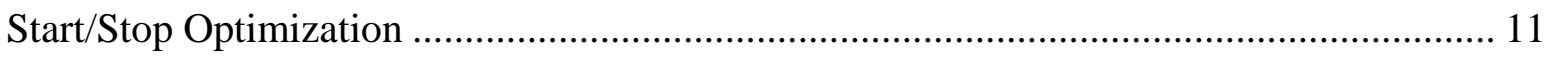

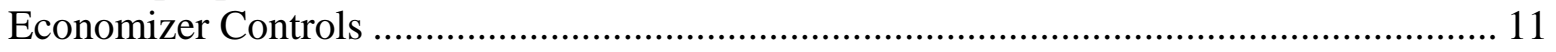

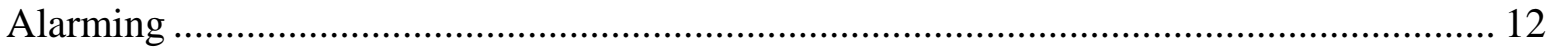

Access to Sensor and Equipment Performance Data ................................................... 13

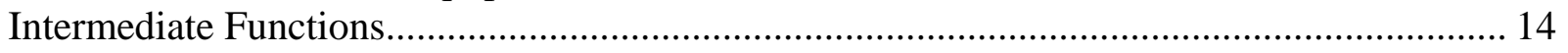

Demand Limiting (Load Shed / Load Rolling)............................................................ 14

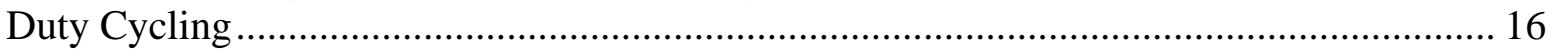

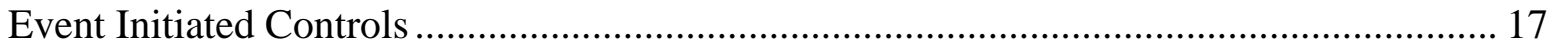

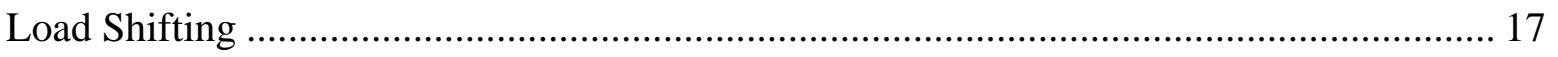

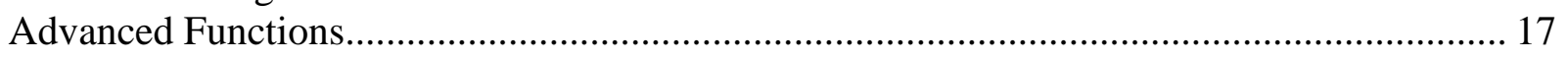

Sequencing and Loading Multiple Chillers/Boilers ................................................... 17

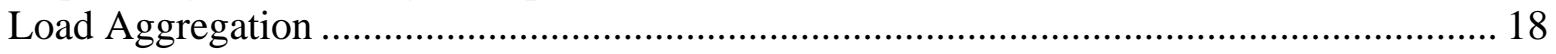

Automated Fault Detection and Diagnostics ......................................................... 18

Monitoring Simultaneous Heating and Cooling .......................................................... 19

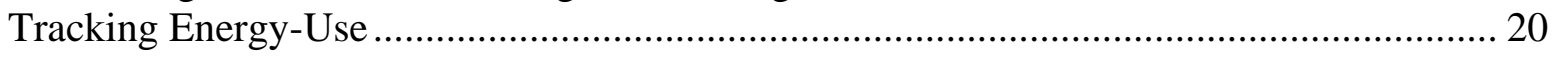

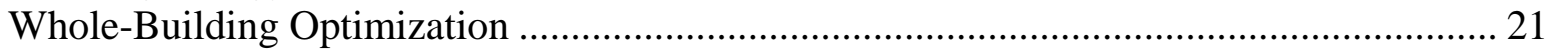

Whole-Site Optimization, Integration plus Security .................................................. 21

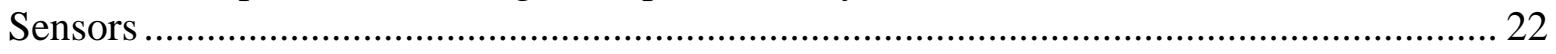

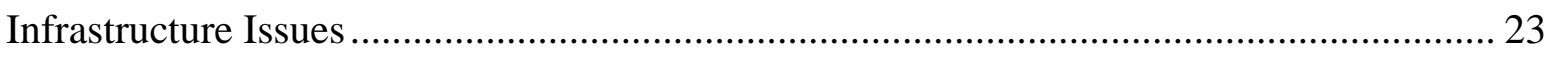

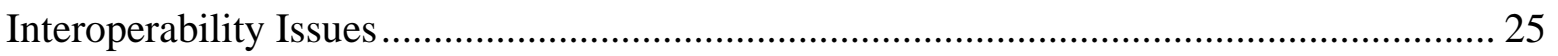

Integration of HVAC Systems with EMCS ......................................................... 27

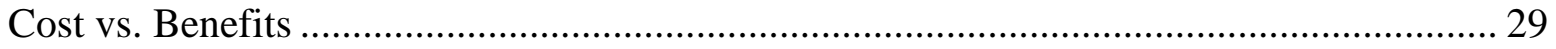

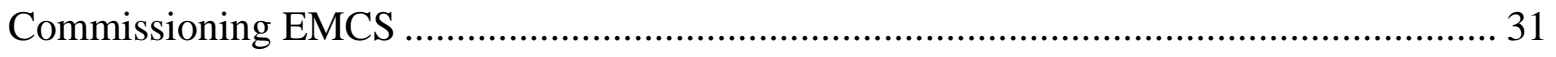

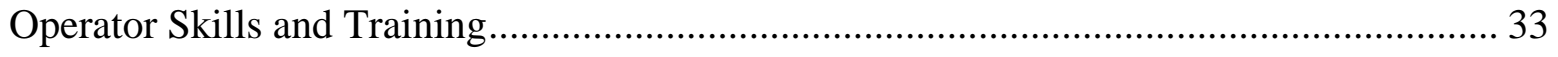

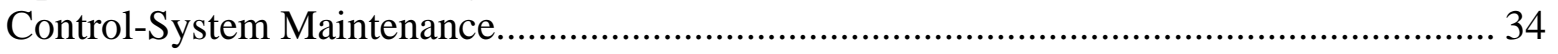




\section{Figures}

Figure 1. Schematic of an Example Control Loop .......................................................... 3

\section{Tables}

Table 1 - Summary of Desired EMCS Functions and Capabilities.......................................... 7 


\section{Introduction}

Energy management and control system (EMCS) technology has evolved over the past 3 decades from pneumatic and mechanical devices to direct digital controls (DDC) or computer based controllers and systems. Today's EMCS systems consist of electronic devices with microprocessors and communication capabilities. Widespread use of powerful, low-cost microprocessors, use of standard cabling, and the adoption of standard communication protocols (such as BACnet, LonWorks, and MODBUS) have led to today's improved EMCS systems. Most modern EMCS systems have powerful microprocessors in the field panels and controllers that will soon be embedded in sensors as well. Therefore, in addition to providing better functionality at a lower cost, these EMCS systems also allow for distributing the processing and control functions to the field panels and controllers without having to rely on a central supervisory controller for all functions. Although true interoperability is still myth rather than a reality (as discussed later in this report), a new EMCS installation should consider standard protocols (such as: BACnet, LonWorks, and MODBUS, etc.), web-based or web-enabled controllers, and control networks that can be integrated with the internet and intranets.

The open protocol question has to be answered with a great deal of thought and engineering. Currently BACnet and LonWorks are the two most popular, truly open protocols for use in communications between man-machine interface (MMI) or front-ends and lower tiered controllers. MODBUS is used widely in the Supervisory Control and Data Acquisition (SCADA) or utility side of systems. If you want to integrate your waste and water treatment plants along with utility electrical monitoring, then MODBUS can be used to bring these operations on to the master site EMCS operation. This then allows for far better control and integrated operations between different divisions into one shared system. When determining whether to go to an open or closed protocol, one must look at the actual application and where it will be applied. Open protocols allow for more than one vendor to talk to a master controller and share basic point data only. It does not allow for coding and programming functions to take place between different vendors. You can not use vendor A's software to program vendor B's controller. You must use each vendor's software and proprietary tools to program or change the program within a controller. This option does not apply to front-ends or MMIs. Some front ends or operator work stations can share data using dynamic data exchange (DDE), OPC or other information sharing protocol. You will generally not want more than one MMI or front-end on a site because of training and operational requirements. Another option, if you are working with newer systems, is web-based systems. These systems can use standard web browsers to view pages of data. They are currently more limited in programming and capabilities than older systems. They do have a place in some installations and should be considered. Higher security concerns play a part when you start to apply a web-based system. Examples of a good application for BACnet might be a Trane chiller interface to an EMCS system. A poor example would be to take an existing building and add one or two BACnet devices into an operating proprietary system. Also, training must be weighted into the decision. If you perform software maintenance in house, then more than one vendors training may be required, if you purchase from more than one vendor. 
When a new EMCS installation is being considered or a replacement of an existing EMCS is being planned, a detailed plan is essential. The plan should address the following items (extracted with some modifications from http://www.hpac.com/microsites/pdf/santos_0105.pdf):

1. Current Installed EMCS System
a. Hardware
b. Work stations, operator interfaces
c. Communication
d. Interoperability
e. Salvageable pneumatic or conduit infrastructure

2. Planned Installations

3. Analysis
a. Cost issues
b. Procurement/contracting limitations and requirements
c. Standard/open protocol considerations
d. Accuracy requirements/types of applications
e. Current use of system
f. Future needs
g. Training needs
h. Commissioning needs

4. Internal Issues/Perspectives

5. Capabilities of Local Control Vendors
a. Products
b. Resources (qualified people, response times)

6. Need for Standards
a. Specifications
b. Sequences

7. Existing Base EMCS Infrastructure and Compatibility.

This report lists the functions and capabilities that are desired in an EMCS and provides a brief description of each of the desired functions. In addition, it also provides a brief discussion of the following topics: sensors, infrastructure, interoperability, EMCS integration of heating, ventilation and air conditioning (HVAC) equipment controls, security, commissioning of EMCS, and operator training and maintenance issues. 


\section{Functions and Capabilities of Energy Management Control Systems (EMCS)}

An EMCS may be known by various other names such as: building automation system (BAS), building management system (BMS), energy management system (EMS), and facility management system (FMS). For this document, the EMCS is explicitly defined as the sensors, transmitters, data acquisition, and data processing performed at the user (building) level as well as data and control systems that are more global to full campus control schemes. The EMCS may also have a global supervisory controller to perform high-level tasks (e.g., resetting temperature setpoints based on building conditions and scheduling on/off times). The hardware generally consists of a distributed computer environment with smart controllers strategically located throughout the system (buildings and base-wide network). Several network terminals or PC computers can be attached to these networks to provide a man-machine interface (MMI).

There are a wide variety of functions an EMCS can perform, depending on its capabilities. At a minimum, the EMCS has a sensor(s) to measure a control variable(s) (e.g., temperature and flow rates), a controller with the capability to perform logical operations and produce control outputs, and a controlled device(s) that accepts the control signals and perform actions (e.g., move dampers and valves), as shown in Figure 1.

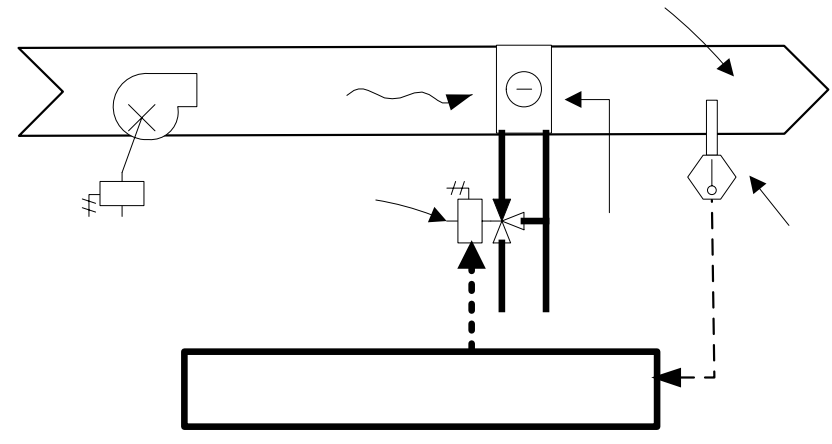

Figure 1. Schematic of an Example Control Loop

Interface systems are used to integrate to lower level packaged components. EMCS modules of this type are able to "integrate" or connect and communicate to facility plant equipment, which has microprocessor-based controls. The different vendor types of equipment that these modules can connect to include: boilers, chillers, variable speed drives, power metering equipment, electrical switchgear/uninterruptible power supply (UPS), etc. and other packaged HVAC equipment, which are microprocessor-based. These interfaces give a global management strategy that encompasses entire building and site complexes. 
Main control panels consisting of local area network (LAN) interfaces and lower level communications should be strategically located in buildings throughout the facility. These modules will allow for direct communication to laptop computers and support of all lower level functions. The software should be developed to take advantage of the Microsoft "Windows" operating environment, whereby the users can open multiple windows so that they can access and manipulate mass quantities of data. "Windows" features should be incorporated into the system design, such as mouse point-and-click operations.

\section{Central Information Processing and Control Workstations}

The operator workstations for an EMCS are normally desktop, medium to high end style Pentium or better and should be "Microsoft Windows" based PCs. The workstations should be connected directly into the LAN or directly to any main control panel. A radio-networked laptop PC in an operator truck can also be linked via a radio modem to provide full access to the systems for mobile maintenance and operations staff.

\section{Day-to-Day Operations}

The day-to-day operations and control of environmental conditions (temperature, humidity, air flow) enable the facility managers to have remote, unrestricted access to environmental information on each building or piece of equipment that is part of the EMCS. This access allows for remote and immediate diagnostics and troubleshooting of system conditions. Buildings with EMCS direct digital controls incorporated into the design allow for adjustment of: temperatures, humidity, supply and exhaust duct static pressures and air flows, equipment run times, special condition monitoring, and other environmental conditions that are incorporated into the EMCS design for that particular building.

Most facilities with EMCS controls are designed with automatic recovery mechanical and electrical systems to start backup equipment in case of a fault in normal operations. This happens without operator actions and at the instance of failure. In most instances, automatic recovery prevents faults from interfering with the day-to-day operations. These events happen in the background, and alarms and notifications are made without impact on the facility. These automatic control features are the best business investment of the EMCS system and are invaluable when compared to down time, reporting cost, or environmental accidents. Facilities can also be designed to be fully, remotely controlled and monitored in case of an event in a building causing evacuation. This limits the exposure of personnel during a hazardous event.

EMCS is not a new concept for Department of Defense (DOD) activities. There have been a variety of disjointed efforts to install EMCS at most facilities since the 1970s. Problems experienced with the various systems installed include:

- Improper designs and improper applications of EMCS: A properly designed EMCS requires specialized design knowledge and experience. 
- Faulty installation, commissioning and government checkout of systems: Familiarity with EMCS systems and equipment operations along with building types are required to ensure a system is properly installed and operates as intended.

- Incompatible EMCS systems. EMCS system A can't communicate with system B that doesn’t communicate with system C, etc.

- Inability to use the variety of disparate systems effectively, if at all, for energy and maintenance savings and improved facility environmental conditions: Learning, and fully utilizing one system is difficult; learning, and fully utilizing a multitude of systems is difficult, if not impossible.

- Lack of maintenance on EMCS equipment and systems. Poor maintenance is often a result of a lack of familiarity with the variety of systems installed, as well as logistical problems associated with maintaining a multitude of systems.

- Lack of EMCS training for HVAC technicians or other operators: Lack of training is often caused by multiplicity of system types and technologies.

A note here about an old control saying "A control system will deteriorate down to the level of the least trained technician." Basically what this means is if your technicians are not trained in maintenance of the EMCS system, they will use what they know (jumpers and overrides) to get systems to work. In a short time, the system will not be controlling as designed, and complaints will actually rise from tenants. A well tuned EMCS system can eliminate complaints by more than $75 \%$ over a system that has been bypassed.

A common sense, root-cause analysis of repeated occurrences of these problems provides practical solutions. To have a successful EMCS implementation, the following is required:

- A core group of people having the requisite technical knowledge and experience to ensure proper applications and designs, proper installation and acceptance, proper maintenance and operation, and proper training: A project-working group provides the perfect organizational structure for establishing this core group of people. Normally a very small group, maybe only one staff member, but it is one who takes ownership in the campus-wide system to bring it all together and make it work.

- A campus-wide approach to EMCS with a common communication protocol, common human interface and common system interfaces to achieve compatibility among all connected EMCS devices: The system needs to be designed to match the campus requirements and not just a building's internal HVAC needs.

- The EMCS core group must ensure that the area-wide EMCS system is maintained and operated as intended: One or two staff members need to be fully trained and work daily with the system to keep improving and recommissioning it to ensure optimal seasonal operations. This is usually best performed by staff personnel versus a subcontractor. 
- The EMCS core group must ensure that HVAC technicians and other operators are trained and become specialists on effective utilization of the area-wide EMCS system: Training and retraining are a daily part of an EMCS specialist's tasks. As changes and upgrades are made to the system to optimize operations, these changes need to be disseminated to operations and maintenance staff.

- All appropriate recurring maintenance job descriptions should be rewritten to reflect revised maintenance requirements and associated reduced costs resulting from EMCS monitoring and control.

- Normally, no more than two EMCS vendors on the campus: Any more than two and training falls short of needs. The system that the staff is least trained on generally will deteriorate to a non-operational condition.

The desired energy management and control functions can be broadly classified into three groups: 1) Basic, 2) Intermediate, and 3) Advanced. Basic functions, commonly available, are highly recommended for efficient operations. Most Basic and some Intermediate energy management functions are typically found in many of the EMCS systems available on the market today. Most Advanced functions are not currently available in EMCS systems. Advanced functions, although highly desirable, have not been a high priority of the building control manufacturers. Much of the work to develop Advanced functions has been done by National Laboratories and universities.

Intermediate function control logic is not generally provided nor implemented in most EMCS systems. However, the fundamental building blocks are provided, requiring in-house or thirdparty development, implementation and integration. Also, most functions in the Intermediate group are required only if demand costs are greater than $20 \%$ of the total energy cost.

Advanced functions are currently not generally supported by EMCS systems and are unlikely to be supported in the near future (the next 5 years). Like functions in the Intermediate group, functions in the Advanced group require in-house or third-party development, implementation and integration.

The desired functions and capabilities are summarized in Table 1. 


\section{Basic Functions}

Functions in the Basic group include a minimum set that is required to operate a building: scheduling, chilled and hot-water reset, supply-air temperature reset, thermostat controls, startstop time optimization, economizer controls, alarming, and the ability to seamlessly export sensor and equipment performance data to third-party software applications.

\section{Scheduling Function}

The single most important function of an EMCS is the ability to schedule the operation of major equipment (e.g., chillers, boilers, packaged air-conditioners, heat pumps, and lights). A building that is not occupied 24-hours per day does not require comfort conditioning, ventilation and comfort lighting during unoccupied periods. This saves not only energy, but also unnecessary wear and tear on equipment leading to unplanned maintenance costs. The goal is to have as much maintenance planned, hopefully anticipated just before failure, signaled by control system data. This results in the added bonus of extending facility equipment life. The result in dollar savings to the customer is both immediate and long term. Inability to properly control major equipment or end-uses during unoccupied periods can increase energy consumption significantly (by 15\% or higher) depending on the lengths of the occupied and unoccupied periods.

In areas with high humidity levels during parts of the year, limited operations at night might be required. Indoor sensors will be required to know when the system is getting near dew points and water may start to condense onto walls or colder objects. During dryer times, systems can remain off.

To handle special events, if a building is occupied beyond the normal operating hours, many EMCS systems provide a temporary scheduling or override feature to disable the normally scheduled operation. If the override feature is enabled, it should be implemented in such a way that it is temporary, i.e., the schedule automatically reverts back to normal operations within a few hours or the next day. If the override is not temporary or does not have the ability to reset itself the following day, the major equipment in the building will operate during unoccupied periods, wasting energy. If the system will not revert back to normal automatically, then a procedure should be in place to remind operations to release all overrides when appropriate.

The scheduling functions must allow programming of special schedules for weekends, holidays, and daylight savings time. The equipment and end-uses that benefit from scheduling include: ventilation, exhaust and supply fans (interior and exterior) and security lighting, packaged HVAC equipment, chillers, boilers, air-handling equipment and other miscellaneous HVAC equipment. Some of this equipment can be off during the day and only on at night or during required use times. Most would be off when the facility is unoccupied

Recommendation: The scheduling function is one of the most important functions in operating the building efficiently, and therefore, is highly recommended. 


\section{Chilled-Water Reset Function}

Typically, the supply chilled-water temperature is held constant between $38^{\circ} \mathrm{F}$ and $44^{\circ} \mathrm{F}$, which is acceptable for full-load or near full-load operation. Chillers, however, usually operate at partload conditions for significant periods of time. Resetting the supply chilled-water temperature to match the actual building-cooling load leads to energy savings. Generally there is a $2 \%$ efficiency increase for each degree raised. The supply temperature can be reset based on the outdoor-air temperature, i.e., the supply temperature can be increased as the outdoor-air temperature decreases. Alternately, the optimum supply chilled water temperature can be computed based on the current conditions of the valves in the air-handling units (AHUs) and the supply-air temperature. The implementation details are provided in the 2003 ASHRAE Handbook for HVAC Applications (Chapter 41) ${ }^{1}$. In addition to decreasing chiller energy use, resetting the supply chilled-water temperature will usually reduce the amount of energy used for reheat for a multi-zone environment. In humid climates, the supply chilled-water temperature should not be raised significantly or should be interlocked with humidity control. Although the actual upper bound for the chilled-water should be based on the latent cooling capacity requirements and other criteria outlined in the ASHRAE Handbook, a general rule-of-thumb in humid climates (e.g., mid-west and southern U.S.) is approximately $50^{\circ} \mathrm{F}$. In dry climates (e.g., western U.S.) the temperature can be slightly higher $\left(55^{\circ} \mathrm{F}\right)$.

While using reset control strategies, the use of proper sampling intervals is critical to avoiding oscillation and instability in control. The general rule of thumb is that the sampling interval between reset commands should be greater than the settling time of the control loop. If you have cascading resets (i.e. both the supply chilled-water and supply-air temperatures are being reset), the sampling time between reset commands should be greater than the settling time of the slowest control loop. Savings from resetting the supply chilled-water temperature can range from $\underline{5 \%}$ to $20 \%$, as per the 2003 Handbook for HVAC Applications, Chapter 41, page 41.7.

Recommendation: Because resetting (increasing) chilled-water temperatures during part-load conditions can save cooling energy use, this function is recommended.

\section{Hot-Water Reset Function}

Like supply chilled-water temperature, the supply hot-water temperature can be reset to reduce energy consumption when the boiler is operating at a part-load condition. Besides reducing the energy used to meet the load, another benefit from resetting the hot-water temperature is a reduction in the standby losses of the boiler and piping systems. To eliminate boiler damage from acid formation, a minimum reset temperature should be implemented. As stated in the ASHRAE, 2003 Handbook of HVAC Application, Chapter 41, page 41.5, "To minimize the condensation of flue gases and consequent damage to the boiler from acid formation, however, the hot-water temperature should not be reset below the minimum temperatures recommended by the boiler manufacturer (typically $140^{\circ} \mathrm{F}$ ). Similarly, energy can be saved in steam heating systems by maintaining supply pressure at the minimum level required to satisfy the largest heating load".

\footnotetext{
${ }^{1} 2003$ ASHRAE Handbook of HVAC Application, American Society of Heating Refrigerating and AirConditioning Engineers, Inc., Atlanta, Georgia, Chapter 41 p. 41.1 - 41.39.
} 
Recommendation: Because resetting (decreasing) hot-water temperature during part-load conditions can save heating energy this function is recommended.

\section{Supply-Air Temperature Reset Function}

For constant-air volume (CAV) air-handling units, the supply-air temperature can be reset based on the zone conditions. When the zones are at a "light" load condition", resetting the supply-air temperature will reduce the cooling and reheat energy consumption. Typically, the supply-air temperature on variable-air volume (VAV) systems is held constant because the volume of the air is modulated to meet the zone load. Recent studies, however, have shown that the supply-air can be reset for VAV systems as well, although the controls sequence is more complex.

Recommendation: Resetting supply-air temperature during part-load conditions can save energy; therefore, this function is recommended for constant-air volume systems. For a variableair-volume system, there is tradeoff between the fan and the cooling/heating energy. Also, the control algorithms are more complex. This procedure is not recommended with variable-airvolume systems unless a thorough site-specific analysis is done and the results are favorable.

\section{Static Pressure Reset Function}

For variable-air volume (VAV) air-handling units, the supply-air static pressure can be reset based on the zone conditions. When the zones are at a "light" load condition, resetting the supply-air static pressure will reduce the cooling and reheat energy consumption. Typically, the supply-air pressure on VAV systems is held constant because the volume of the air is modulated to meet the zone load. Recent studies, however, have shown that the static reset based on the zones with the highest damper conditions can save large amounts of energy. Some systems have been seen to run as low as 0.25 inches at lighter loads compared to a 1 to 2 inch normal setpoint.

Recommendation: Resetting supply-air static during part-load conditions can save energy; therefore, this function is recommended for variable-air volume systems.

\section{Thermostat Controls}

Thermostats are used to control the comfort conditions. In commercial buildings, a typical HVAC zone has a number of sub-zones (rooms) all served by a single heating or cooling unit, which is controlled by a single thermostat located in only one of the sub-zones. If the internal loads (e.g., equipment in the space, number of occupants, etc.) and external envelope loads (i.e., heat gain or loss through exterior walls) are uniform across all rooms in a single HVAC zone, a single thermostat is adequate to maintain the comfort across rooms. In many cases, however, the loads are not uniform across the zone. One way to avoid hot or cold spots is to install a number

\footnotetext{
${ }^{2}$ Light load conditions can be identified two ways: 1) by monitoring the return-air temperature from all zones (if return-air temperatures are close to supply-air temperature, the zone load is small); or 2) by monitoring the control output of each of the terminal units.
} 
of temperature sensors (wireless or wired) across the zone (e.g., one in each room) and then to use the average value of the sensed temperatures in the zone to control the HVAC equipment.

It is important to have the ability to reset the thermostats remotely from the central EMCS work station. This will allow for temperature setup during unoccupied summer periods and temperature setback during unoccupied winter periods. It will also provide a mechanism to shed loads during supply shortages or during demand-limiting periods. The ability to setup and setback thermostats reduces energy consumption of supply fans, packaged equipment, chillers, boilers, and air handlers.

Recommendation: Thermostat controls with remote monitoring and setpoint control that are integrated with EMCS systems are highly recommended.

\section{Start/Stop Optimization}

Start/stop optimization or temperature/time optimization, as it is sometimes referred to, is an improvement over simple setup/setbacks. Setup/setbacks are based on time-of-day, while the start/stop optimization strategy accounts for outdoor-air conditions and can include thermal storage of building mass to determine when to startup and setback the HVAC system operation. The optimized stop time permits certain HVAC systems (e.g., chillers and boilers) to be shutdown before the end of the occupied period (e.g., 10 to 15 minutes) and allows the zone temperature to float within acceptable comfort levels. Similarly, the optimized start time permits certain HVAC systems (e.g., boilers and chillers) to start just in time to allow for the zone conditions to reach the acceptable range just when the zone begins to be occupied.

Recommendation: Optimum start/stop function is desirable where buildings are not occupied 24-hours. Even in buildings operated 24 hours a day, 7 days a week, there are generally several time zones that can be shut off to reduce energy usage during some hours of the day.

\section{Economizer Controls}

In cooling mode, there are a number of hours when the outside air is cooler than the return-air stream. Use of "free" cooling when outside conditions are favorable is referred to as airside economizing. An economizer cycle can be employed on built-up AHUs as well as on packaged unitary products, generally referred to as roof top units. For built-up AHUs, the EMCS must provide the necessary sequence of operations for the economizer cycle, while most packaged units come equipped with economizer cycles and built-in economizer controls.

The cooling energy savings from use of an economizer cycle range from $15 \%$ to $50 \%$; the precise savings depending on the type of economizer cycle employed and the climate location. The most commonly used economizer control strategies are: 1 . differential dry-bulb temperaturebased, 2. differential enthalpy-based, 3. high-limit dry-bulb temperature-based, and, 4. high-limit enthalpy-based.

With differential control strategies, the outside-air condition is compared with the return-air condition. As long as the outside-air condition is more favorable, outside air is used to meet all 
or part of the cooling demand. An example of a favorable condition with dry-bulb temperature control would be the outside-air dry-bulb temperature being less than the return-air temperature. If the outside air alone cannot satisfy the cooling demand, mechanical cooling is used to provide the remainder of the cooling load.

With high-limit control strategies, the outside-air condition is compared to a single setpoint or fixed setpoint, usually referred to as a high limit. If the outside-air condition is below the setpoint, then outside air is used to meet all or part of the cooling demand; the remainder of the cooling load is provided by mechanical cooling.

Although the sequence of operations for economizer control appears to be simple, the failure rate in the field is very high. A faulty or improperly operating economizer cycle is difficult to detect because the heating/cooling system generally compensates for the failure by providing more mechanical heating or cooling, and no occupant discomfort is noticed.

Recommendation: Properly operating economizer controls can save significant cooling energy consumption and are highly recommended. In dry climates (e.g. most of western U.S.), dry-bulb differential based economizer controls are preferred, while the enthalpy-based controls are preferred in humid climates (e.g., most of the mid-west and southern U.S.). In either case, comparing the return-air conditions to the outside-air conditions (differential control) has significant gains over the older high limit setpoint type controls (high limit control). This control algorithm also needs to coordinate with the heating and cooling controls.

\section{Alarming}

EMCS provides the function of alarm annunciation and dissemination for the systems currently connected directly to a central location. This provides early detection of trouble/critical alarms during normal and off-shift hours. During off-shift hours, when manpower is greatly decreased, the value of EMCS alarm notification and central location relay and response cannot be underestimated.

The immediate benefit to users include the effective monitoring of critical buildings and utility functions, which is a necessary ingredient to the total operation of the individual buildings and the entire campus as a whole. This ensures that the investment in each of the buildings is protected and not compromised by enabling an immediate and quick response to any building or utility failure. Alarms are useful in detecting many abnormal conditions in buildings. For example, the ability to monitor the space temperature and create an alarm when the conditions deviate from the acceptable range can be useful in mitigating problems before the occupants complain. All modern EMCS systems provide such alarming functions.

Recommendation: Alarms are strongly recommended. They are useful in both day-to-day operations and the maintenance of buildings and facilities. The receiving alarm computer or station does not have to be manned if alarms are transmitted automatically via pager or email to the appropriate staff. 


\section{Access to Sensor and Equipment Performance Data}

Having an EMCS in every building is not sufficient to address the growing needs of the facility manager and operator. Many of the intermediate and advanced functions, described in the following section, are generally not available in most EMCS systems. They have to be developed and integrated in-house or by a third-party software provider because EMCS vendors are unlikely to provide this functionality in the near future. Keys for successful implementation of these functions is easy access to sensor, controller and equipment performance data distributed throughout the facility on a control network managed by EMCS and networks that tie the EMCS with the rest of the enterprise (internet or intranet). This will lead to the next generation of distributed facilities management systems. Without easy access to data, it would be difficult to realize all the benefits of distributed facilities management. These benefits include:

- Lower energy expenses,

- Fewer occupant complaints and faster resolution of problems,

- Reduced liability and litigation expenses, relating to indoor air quality (IAQ) issues,

- Improved performance of occupants because of a better indoor environment.

Although the details of data gathering depend on the type of EMCS and the protocols it supports, most EMCS systems provide some standard methods to access data from geographically distributed facilities. Many EMCS manufacturers provide DDE/OLE Direct Data Exchange/Object Link and Embedded servers to facilitate data exchange between controllers/devices and software application programs. Having an EMCS that supports a standard data exchange method (e.g., DDE, OLE, BACnet, LonTalk) does not guarantee success. For example, a third-party application that can import data from one EMCS using a standard data exchange method might not work with another EMCS, although both EMCS systems support the same standard.

Most EMCS manufacturers support trend logging for sensor, controller and equipment data, which are generally not in a standard format. Although trend logs are useful, they require some pre-processing before they can be directly used with third-party applications. In addition, the logs are generally not available in real-time, and it is difficult to automate functions using trended data. Therefore, trend logs should not be confused with real-time direct access to sensor, controller, and equipment data through use of standard communication or data exchange protocols (DDE/OLE, BACnet or LonWorks).

Recommendation: The ability to extract data is essential for implementation of functions that are not directly supported by the EMCS. Therefore, only consider those EMCS systems that support standard data exchange methods (DDE/OLE or BACnet). These functions are critical if the EMCS system is planned to gather meter, run time or other data to import into another software package. These might be maintenance request tracking packages or utility monitoring packages. 


\section{Intermediate Functions}

Functions that fall in the intermediate group include: demand limiting (load shedding or load rolling), load shifting, duty cycling, event initiated controls and monitoring simultaneous heating and cooling.

\section{Demand Limiting (Load Shed / Load Rolling)}

In some regions of the country, demand charges can be a significant fraction of electricity costs. Often utilities have ratchet clauses that affect the billed peak for the next 12 months. Demand limiting is one way to control excessive demand charges. It allows for systematic shedding of electric loads when the peak demand of the building approaches a preset level. Many chillers have demand limiting features that, when triggered, limit the power to the compressor. This can also be implemented in an EMCS. Demand limiting supervisory controls must ensure that the desired demand limit is not exceeded at any time during the month or the season. Some advanced control features, such as building pre-cooling and pre-heating, can help alleviate uncomfortable conditions when AHU or chiller usage is limited during demand limiting periods.

For illustration purposes, consider the following example:

A facility has an EMCS system capable of automatically running an independent load shedding software scheme. The sequence reads the base meter electric current rate data and broadcasts, over the independent network, demand levels for the buildings to follow. Demand levels follow the base utility rate structure for costing demand. The facility rate structure has the following three peak time periods: on-peak, partial-peak and off-peak.

The peaks are defined as follows:

\section{Summer months, May through October:}

1. On-peak: 12 noon thru 6:00 P.M., Monday thru Friday, excluding holidays,

2. Partial-peak: 8:30 A.M. through 12 noon and between 6:00 P.M. through 9:30 P.M., Monday thru Friday, excluding holidays,

3. Off-peak: 9:30 P.M. thru 8:30 A.M., Saturdays, Sundays and all holidays.

Starting at 11 A.M., the EMCS system starts ramping down, so that the systems are able to achieve setpoint at the start of the On-peak time period. During the On-peak time frame, the EMCS systems will control to the On-Peak prescribed kilowatt (kW) demand limit set-point maximum. During the Partial-peak periods the EMCS systems control to the prescribed Partialpeak KW demand setpoint value. All other days and times, the EMCS system will control to the Off-peak prescribed kW maximum setting.

\section{Winter months, November thru April:}

1. On-peak: none

2. Partial-peak, 8:30 A.M. thru 9:30 P.M., Monday thru Friday, excluding holidays,

3. Off-peak: 9:30 P.M thru 8:30 A.M weekdays plus Saturdays, Sundays and all holidays. 
During the partial-peak time frame, the EMCS system will control to the partial-peak kW demand setpoint value. During all other days and times (off-peak times), the EMCS system will control to the off-peak prescribed $\mathrm{kW}$ setting.

Each of the three demand time slots have a corresponding maximum $\mathrm{kW}$ setpoint. These would be "not to exceed" setpoints. For this example:

1. The meter allocation was 12.6 megawatts

2. The not to exceed setpoint for all time frames was 12 megawatts.

The main load shedding software was designed to have 10 separate groups and 4 control stages, as defined below. Buildings were assigned to specific groups, based on priority, with the $1^{\text {st }}$ group being the least important (most capable of withstanding environmental change) and the $10^{\text {th }}$ group being the most important (least capable of withstanding environmental change).

Group 1 executes load shedding first and group 10 executes load shedding last. As the kW level rises because of increased load, the $1^{\text {st }}$ group executes stage 1 load limiting, followed by Group 2, etc. until all groups have activated stage 1 . After all groups have executed stage 1 , and the demand level continues to climb, group 1 would execute stage 2, followed by Group 2, etc., until all groups have executed stage 2, followed by stage 3 and 4, respectively.

Caution, there should be a gradual ramp down of the setpoints prior to on-peak setting to balance the loads as the systems come into alignment.

\section{Master load shed/load roll group settings:}

Target Base Load $\quad 12000 \mathrm{~kW}$

$\begin{array}{lrrrr} & \text { Stage 1 } & \text { Stage 2 } & \text { Stage 3 } & \text { Stage 4 } \\ \text { Group 1 } & 11600 & 11700 & 11800 & 11900 \\ \text { Group 2 } & 11610 & 11710 & 11810 & 11910 \\ \text { Group 3 } & 11620 & 11720 & 11820 & 11920 \\ \text { Group 4 } & 11630 & 11730 & 11830 & 11930 \\ \text { Group 5 } & 11640 & 11740 & 11840 & 11940 \\ \text { Group 6 } & 11650 & 11750 & 11850 & 11950 \\ \text { Group 7 } & 11660 & 11760 & 11860 & 11960 \\ \text { Group 8 } & 11670 & 11770 & 11870 & 11970 \\ \text { Group 9 } & 11680 & 11780 & 11880 & 11980 \\ \text { Group 10 } & 11690 & 11790 & 11890 & 11990\end{array}$

\section{Building Load shedding stage definitions/scheme:}

Each facility shall be assigned a group number. An example would be facility 333 might be assigned to group 6, meaning it would be load shed near the middle of the priority scheme. The 
EMCS system in the building listens for the broadcast of a demand level from the master meter and changes its sequenced control per the following.

DEMAND LEVEL \# 1: Resets T/Stat setpoints out $1^{\circ} \mathrm{F}$ from current setpoints.

DEMAND LEVEL \# 2: Resets T/Stat setpoints out $2^{\circ} \mathrm{F}$ from original setpoint and limits chilled water valves to $75 \%$ of full open capability.

DEMAND LEVEL \# 3: Resets T/Stat setpoints out $4^{\circ} \mathrm{F}$ from setpoint and limits the chilled water valves to $25 \%$ of open capability.

DEMAND LEVEL \# 4: Load sheds / load rolls (often referred to as duty cycling) main chiller or direct expansion $\mathrm{A} / \mathrm{C}$ unit off. Chiller and fans shall be monitored for allowable off and short cycling run times. Once released to run, a unit shall be allowed to run a preset amount of time before cycling off again. Units shall be only allowed to stay off a preset amount of time.

Recommendation: Because this function is useful to reduce peak consumption and control loads base wide, it is recommended when the demand charge is a significant percent of the total energy cost (greater than 20\%) or other drivers require the ability to shut off or reduce peak energy usage. This function is not readily available in most EMCS systems and has to developed and integrated in-house or with the help of a third-party service provider.

\section{Duty Cycling}

Duty cycling is cycling of equipment ON or OFF to control the building peak energy consumption while still maintaining comfort conditions. Duty cycling is one approach to demand limiting and is a means to change or control the duty cycle (i.e., the ratio of on-period to total cycle time) of on/off controlled equipment (e.g., unitary air conditioners, heat pumps, furnaces). There are a number of ways to implement duty cycling, as described in a FEMP Technology Focus article ${ }^{3}$.

The following is extracted from the article:

"In the past, a variety of methods were used to implement duty cycling on heating and cooling equipment. These ranged from simple fixed-time-based strategies to sophisticated optimization methods. In all of these methods it is ultimately the off-period that is either fixed or adjusted in either a given reference period (typically 15 or 30 minutes), or dynamically based on temperature measurements. This results in the imposition of an equipment duty cycle that is primarily under the control of the cycler; i.e., it overrides the "natural" duty cycle of the thermostat."

Recommendation: Because this function is only useful to reduce the peak consumption, it is only recommended when the demand charge is a significant percentage of the total energy cost

${ }^{3}$ FEMP Technology Focus: Duty Cycling Controllers Revisited - May 1998, http://www.eere.energy.gov/femp/pdfs/DutyCycling-TF.pdf 
(greater than 20\%). This function may not be available in most EMCS systems and has to be developed and integrated in-house or with the help of a third-party service provider.

\section{Event Initiated Controls}

Event initiated controls are similar to demand limiting controls where a set of control sequences are initiated when a certain external event occurs. For example, if the facility is participating in an emergency demand response or load curtailment program offered by some utilities, the utility may send a signal directly to the EMCS or a page to the building operator. If the utility sends a message directly to the EMCS, the actions are automatically followed, while the building operator has to initiate the external event if a pager notification is used. Another example is a facility that is normally unoccupied except for special events (e.g., a gymnasium, lyceum or a sport facility) for which control sequences can be event initiated.

Recommendation: Because this function is only useful to reduce peak energy consumption, it is only recommended when the demand charge is a significant percent of the energy cost (greater than 20\%). This function is not readily available in most EMCS systems and has to be developed and integrated in-house or with the help of a third-party service provider.

\section{Load Shifting}

Load shifting is another demand-side management process where the load is shifted from peakconsumption periods to non-peak periods, usually to take advantage of time-of-use rates or realtime pricing. It requires a thorough analysis of the loads and processes that can be shifted and the benefits arising from shifting. Most EMCS systems can be programmed to shift electric loads, but the facility manager or operator must currently perform analysis of what loads to shift and calculate manually how long they can be shifted. Technology should become available to automatically support and execute such decisions over the next several years.

Recommendation: Because this function is only useful to reduce the peak energy consumption, it is only recommended when the demand charge is a significant percent of the total energy cost (greater than 20\%). This function is not readily available in most EMCS systems and has to be developed and integrated in-house or with the help of a third-party service provider.

\section{Advanced Functions}

Functions that fall in the advanced group include: optimal sequencing and loading multiple chillers and boilers, load aggregation, automated remote fault detection and the diagnostics of building systems and subsystems, tracking energy usage and end-uses by building or groups of buildings, and whole-building optimization and optimization across multiple buildings and building systems.

\section{Sequencing and Loading Multiple Chillers/Boilers}

In situations where a facility has multiple chillers, the loading of chillers is generally done based on the cooling load, available capacity and a desire to have even run times for all available units. 
This approach, however, does not necessarily lead to minimum energy consumption. Although the order of bringing chillers online should be based on minimizing the total power consumption, including pumps and cooling tower or condenser fans, consideration should also be given to maintaining even run times where possible. The optimal sequencing of chillers requires the knowledge of part-load performance, not only for the chiller but also pumps and cooling towers, because the goal is to reduce total energy consumption (i.e., chiller, pump, and cooling tower). At loads less than $30 \%$, the energy consumption of pumps and cooling tower fans can be greater than the chiller.

Optimal sequencing of boilers in a multiple boiler system is similar to that of chillers, where the total energy consumption is minimized to meet the required thermal load. The EMCS systems available today do not provide control algorithms to optimally sequence chiller/boilers; these sequences have to be developed in-house.

Recommendation: Currently, EMCS systems do not provide for optimum sequencing of chillers and boilers because algorithms are complex and dependent on the characteristics of all associated equipment: chiller, pumps, cooling tower. Custom code and third-party tools or algorithms should be seriously considered to sequence equipment, if there are multiple boilers/chillers in a plant.

\section{Load Aggregation}

To negotiate favorable utility rates and tariffs, as well as to ensure that the contract limits are not exceeded, the aggregated load and demand profiles of individual buildings and the entire distributed facility are required. Aggregation is facilitated by connecting existing revenue meters or sensor readings as data in real time for subsequent analysis by operators or other building staff. Aggregating and monitoring real-time data across buildings/facilities and simultaneously monitoring the end-uses in each building/facility can help identify where to curtail energy usage if demand is close to exceeding the negotiated limit. Although EMCS generally do not provide this functionality, the existing control network can be leveraged to help aggregate meter readings.

Recommendation: Currently, EMCS systems do not provide a function to aggregate and monitor loads across buildings/facilities. Because this function is only useful to manage building/facility peak energy consumption, it is only recommended when the demand charge is a significant percent of the total energy cost (greater than 20\%). This function has to be developed and integrated in-house or with the help of a third-party service provider.

\section{Automated Fault Detection and Diagnostics}

Effective use of building system diagnostics can help facility managers and operators cut the cost of operations and reduce consumption of resources while improving the comfort and the safety of the occupants. Continuous diagnostics for building systems and equipment will help remedy many problems associated with inefficient operation of buildings by automatically and continuously detecting system performance problems and bringing them to the attention of building operators. Some of these problems (e.g., simultaneous heating and cooling) might 
otherwise go undetected. Advanced diagnostic tools can even suggest causes of problems, make recommendations for solving problems, and estimate the cost of not solving a problem.

Although fault detection and diagnostics have been an active research topic for several years, only a few software applications are available today. Lack of a well-defined infrastructure and difficulties in accessing sufficient quantity and quality of data are two of the reasons for the slow start. Control manufacturers have not yet started packaging diagnostic tools with EMCS systems. At the present time, these diagnostic tools have to be used as standalone third-party applications.

Recommendation: Currently, EMCS systems do not provide functions to detect and diagnose faults. It is unlikely that they would do so within 5 years. However, having automated fault detection and diagnostic capabilities can enhance the operation and maintenance of a facility. Although a number of researchers have been working in this general area, there are not many "commercial" quality tools available currently. Existing tools should be evaluated and used where possible and additional capabilities should be developed and integrated with the help of a third-party service provider as needed.

\section{Monitoring Simultaneous Heating and Cooling}

Simultaneous heating and cooling can occur in a building intentionally or unintentionally. In a multi-zone system, if one of the zones is calling for heating and the rest are calling for cooling, the air to the zone that requires heat will be cooled first and then heated. This generally occurs in systems when cooling is the dominant load and is provided by a central chiller plant. Reheating of the air usually occurs in variable air volume (VAV) or constant air volume (CAV) terminal boxes, where the air temperature is brought to the correct temperature for discharge into each zone. This should be avoided or minimized where possible. Simultaneous heating and cooling can also occur because of faulty air-handling unit (AHU) controls or leaky valves. In most cases, such faulty controls cannot and will not be detected because they do not lead to occupant discomfort. When a leaky hot water valve leaks water into the heating coil, it increases the cooling load with little or no discomfort to the occupant. If the leak is not severe (less than $50 \%$ ), it can go undetected because the cooling coil compensates for the leak. The logic necessary to detect such faulty operations is not generally provided with EMCS systems. Algorithms are under development for automatically detecting these problems, but they may not be included in EMCS systems for several years.

Recommendation: Currently, EMCS systems do not provide for a mechanism to detect simultaneous heating and cooling. Depending on the type and condition of the system, the impact of simultaneous heating and cooling can be significant and difficult to detect. Custom code or third-party tools or algorithms should be seriously considered to mitigate simultaneous heating and cooling from occurring. Managing this function is a requirement of the EMCS specialist, who is responsible for energy usage and the operations of the EMCS system. 


\section{Tracking Energy-Use}

Although tracking energy use for major equipment and end-uses has several benefits, it is not generally done through an EMCS. Leveraging EMCS infrastructure to track energy consumption of major end-uses can be less expensive than setting up standalone systems to collect the end-use consumption data ${ }^{4}$. The benefits of energy end-use tracking for a facility manager of a large campus with distributed facilities include:

- Ability to benchmark historical, normalized (i.e., with respect to weather, size, sales) enduse consumption between similar buildings/facilities. Comparison with benchmarks can help identify buildings with operational inefficiencies.

- Ability to forecast energy budgets and prepare energy purchasing plans

- Ability to track energy savings from performance contracting

- Ability to detect unscheduled operations of equipment as a result of control malfunction, errant programming or to detect controls that have been manually overridden.

Military installations have historically used utility meters to allocate utility usage to tenants and to manage energy usage. Military installations are unique within the federal government in that they normally consist of numerous industrial, administrative, housing and support buildings arrayed in a campus type environment. One or more master meters equipped with time of use demand, energy consumption and power factor meters serve these campuses. Because of the large size of these military complexes, often in excess of a 1000 buildings, it is not cost effective to meter every building. Instead, defense components apply meters at sub-meter points, large utility users or upon the request of tenant commands. Buildings not equipped with meters because of their small energy usage, are allocated usage based upon engineering calculations. In comparison, most other federal agencies' buildings are within small complexes, often housing multiple federal agencies or individual structures. These structures can easily be metered individually, but face the problem of allocating usage across the tenants within the building.

In August 2001, the House of Representatives passed House Bill HR 4 known as, Securing America's Future Act (SAFE) 2001, which contains provisions on requiring electrical metering of all federal buildings. In April 2002, the Senate passed S517, which also requires metering. Congressional intent of these metering requirements is to use the increased awareness of real time utility usage to affect management decisions to promote energy conservation.

The Department of Defense documentation fully supports the use of meters to manage energy usage where it is cost effective. Meters should be installed at locations where the energy usage being monitored justifies the cost of installing, maintaining and reading the meter. This cost benefit analysis can be tempered by the specific needs of the host, such as metering a specific tenant, operational requirements, etc. Where the serving utility company has established interval tariffs, based on time of use, defense components should monitor electrical demand on a

\footnotetext{
${ }^{4}$ The Energy Bill being considered in Congress will require all end-use consumptions greater than $50 \mathrm{~kW}$ to be metered and tracked.
} 
continuous basis. This management can normally be accomplished through software resident on an automated meter reading system (AMR), supervisory control and data acquisition (SCADA) system or Energy Monitoring and Control System (EMCS). In all cases, technology improvements and personnel constraints dictate maximum use of remote meter reading.

Recommendation: Currently, EMCS systems do not provide a function to track energy use within equipment or buildings. If the provisions in the Energy Bill being considered in the Congress become law, there will be a need to track end-uses; otherwise energy tracking should be considered on a site-by-site basis.

\section{Whole-Building Optimization}

In the deregulated utility environment, one of the greatest cost-savings opportunities for facility managers and operators lies in the ability to control and optimize whole-facility energy consumption. Some utilities are now offering rates that vary by hour-of-day and day-of-week, similar to the real-time pricing rates and time-of-use rates offered by some utilities. To take advantage of time-varying rates, facilities will need advanced control strategies. These strategies include HVAC load shedding for chillers, thermal storage, supply and zone temperatures, fans, and pumps; load shifting, using pre-cooling or thermal storage; and fuel shifting for gas, oil, and standby generators. These strategies not only require monitoring the performance of equipment and accessing data from sensors and meters but also the capability of controlling equipment from a central location.

In regulated areas, in addition to the energy charge, most utilities also have a demand charge that can be as high as $\$ 25$ per $\mathrm{kW}$ or more. In some cases, the peak period is tied to the utility's system-wide peak. In such a case, predicting the system-wide peak and implementing the loadcontrol measures during a 2 or 3 hour window surrounding the anticipated time of the peak will save a large facility (such as a military base) a significant amount in demand charges. Such advanced control and campus wide optimization tools are still in their infancies, so the EMCS vendors are unlikely to provide them in the near future.

Recommendation: Currently, EMCS systems do not provide this functionality, and it is unlikely that they would do this in the near future. The need of this function should be evaluated on a site-by-site basis because it requires a significant in-house or third-party development effort.

\section{Whole-Site Optimization, Integration plus Security}

In the current information technology (IT) environment many computerized systems are being advertised to directly connect to the facility local area network (LAN). This is true of all EMCS systems. Several issues have to be addressed before connecting an EMCS system to a LAN.

Security of the LAN is primary. If connecting to an open base LAN system, the base IT operations staff will have security requirements that will need to be addressed and followed. Access to the EMCS system will be restricted to these facility LAN requirements. Most of these will have to do with outside world connections like modems, Internet access and types of software running on connected computers. Most front ends for EMCS systems will run on 
Windows platforms or Windows-based operating systems. The equipment in the field normally will have a small vendor operating system and is generally not subject to attack from normal outside means. Front ends will have to be hardened against attacks with fire walls and virus protection software. Because most viruses and disruptions come in via email functions, it is not recommended that email accounts and EMCS software be operational on the same critical front end computers. Alarms and base-wide control schemes are critical to operations of a complete EMCS system. If the LAN is being overloaded for any reason, these alarms may not make it to their intended recipient in a timely manner. This can be another drawback of having the EMCS on a base LAN system.

Phone-in ability is a very good maintenance tool for the support of any EMCS system. Depending on software, encryption and access requirements, these connections can be setup for remote, outside service from vendors or base personnel.

Recommendation: It is recommended that base EMCS systems be connected to an independent LAN on the base. This can be either a true standalone network or a logical network that isolates it from other network problems and outages. If the system is on a standalone network, then phone-in support becomes a much easier and secure option to implement. A standalone network also opens up the possibility of web access.

\section{Sensors}

For years, the use of sensors in facilities and buildings has been limited by their installation costs. Although the functionality of EMCS systems has increased significantly over the past three decades, control vendors still only install the most rudimentary sensors in buildings to control construction costs. The unfortunate consequence is that the controls that can be provided, the tools to identify operational problems and diagnose them, and the information required to provide energy and other performance metrics to building staff, is often limited because the necessary data are not available. Many of the intermediate and advanced functions that are described in this report require additional sensors. Benefits of having additional sensors far outweigh the installed cost of sensors. Wireless sensors should soon begin making their way into building controls, and the cost of installing additional wireless sensors in existing buildings will be significantly lower installation cost than wired sensors.

Recommendation: Careful evaluation of information needs should be performed when faced with EMCS upgrades or new EMCS installations. The addition of a few key sensors in each building may have a significant impact on the usefulness of collected and monitored data for diagnostic purposes. It may require a third party evaluation to determine additional sensor needs. Wherever possible, consider installing additional sensors. A minimal set of sensors will be installed for a particular control scheme. It is recommended that additional sensors be installed to facilitate remote monitoring and diagnostics for facility maintenance personnel. These additional sensors pay back in weeks by eliminating wasted trips to the field to verify the operational status of systems. Some sensors not normally installed but recommended are sensors to monitor return air temperature, temperature between heating and cooling coils, reheat coil discharge temperature, pump suction and pump discharge pressure. 


\section{Infrastructure Issues}

In addition to increased functionality and distributed processing capabilities of the EMCS, recent developments in open communication standards, as well as the pervasive use of internet and intranet technologies, have created a flood of new options for owners and operators of distributed facilities. A number of control manufacturers are supporting standard protocols and an ability to integrate control networks with local area networks (LANs - intranet) and wide area networks (WANs - internet).

There are several benefits from integrating control networks with LANs and WANs. A number of Intermediate and Advanced EMCS functions rely on the ability to remotely monitor and control distributed facilities efficiently. To benefit from these changes, facility managers, owners, operators, and energy service providers are challenged to acquire or develop new capabilities and resources to better manage building information and, in the end, their buildings and facilities.

Although integration of control networks with LANs and WANs provides the ability to better manage distributed facilities, it requires additional security measures. The use of fire-walls and other security technologies (e.g., virtual private networks) can provide the necessary security. In many installations the control network is only exposed to the LAN. This restricts access to the control network to only those computers that are on the LAN, provided that there is a fire-wall that prevents internet traffic from accessing the LAN while still providing a mechanism for access via the LAN by authorized users for control and facility management. Most control vendors with web-enabled controllers also provide password protection for login and the encryption of data that is transferred off the network, further enhancing security. These capabilities should be explored with each EMCS vendor and the in-house information technology staff. 


\section{Interoperability Issues}

Some of the major challenges facing the facility manager/operator in the installation of an EMCS are configuring, programming, tuning, and commissioning the controllers, sensors, and actuators. This process is not only error-prone and time consuming, but also requires a skilled technician or an engineer. Use of devices, even those that meet standard communication protocols such as BACnet and LonWorks, from different manufacturers further complicates the installation process because configuring and programming similar devices from different vendors require different software tools provided by the manufacturers.

The standard protocols define the functional requirements and methods to share information between devices. However, proprietary programming languages and tools are generally required to configure and program even the standards-compliant devices. Therefore, devices from different manufacturers are seldom used. Furthermore, if a device needs replacement, even when using state-of-art EMCS systems that support standard communication protocols, more often than not, one is forced, by practical considerations, to replace it with a device from the original manufacturer. Although a device from a different manufacturer could have been used at a lower cost, the building operators and owners prefer to use replacement components from the same manufacturer because they are familiar with the configuration and programming requirements of the device. In this sense, most traditional state-of-art systems are not truly open (even those that are based on standard protocols). To be truly open, a device should not only be functionally equivalent and be able to share information with other devices, it should also provide an open configuration interface so that tools made by other manufacturers and software companies can be easily installed and configured. 


\section{Integration of HVAC Systems with EMCS}

Manufacturers of a number of large HVAC systems such as chillers, boilers, and distributed generation equipment, provide their own individual controllers to operate and control the system. Although system controllers provided by the equipment manufacturer will operate in a standalone mode, most often they rely on the EMCS to provide global parameters (setpoints, outdoor air temperatures, schedules).

Recommendation: Before investing in a new EMCS make sure it has the ability to communicate with existing HVAC equipment or vice-versa. 


\section{Cost vs. Benefits}

It is difficult to talk cost vs. benefits, in general terms, because there are many issues that need to be considered. This section will discuss the cost benefits of different operational schemes- and savings calculation methodologies. It includes a definition of terms.

Basic savings “rules of thumb”:

Because some EMCS functions are only useful to reduce peak consumption, it is only recommended to add these functions when the demand charge is a significant percent of the energy cost (greater than 20\%). Engineering evaluations may provide an exception to this.

The cooling energy savings from the use of an economizer cycle ranged from $15 \%$ to $50 \%$, the precise savings depending on the type of economizer cycle employed and the climate location.

Savings from resetting supply chilled-water temperature can range from 5\% to 20\% (2003 Handbook for HVAC Applications, Chapter 41).

Example savings calculation methodology:

After an extensive evaluation of the example facility's energy usage reports, energy bills, and other square footage sources, the following can be calculated from the data:

- $\quad$ Based on the start of the example EMCS Project, FY2000 was used as the baseline year. Facility housing costs were not included.

- The best indicator of energy usage is Btus per square foot per degree-day. This is because the example facility is in constant flux on energy cost, demand charges and even changes in square footage. 


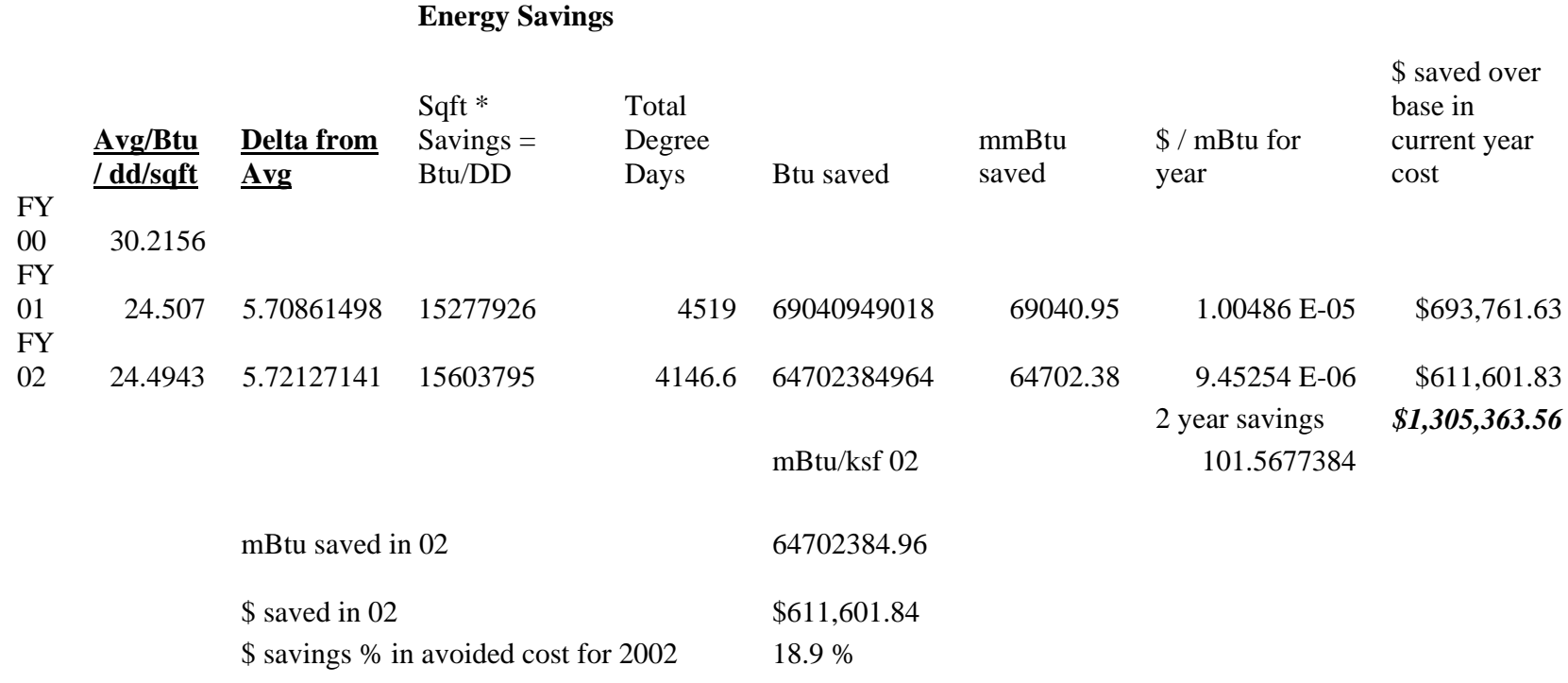

Energy use per square foot went down from 30.22 average Btu/sqft/dd in the base year to 24.49 $\mathrm{Btu} / \mathrm{sqft} / \mathrm{dd}$ in 2002. This is a reduction of $5.72 \mathrm{Btu} / \mathrm{sqft} / \mathrm{dd}$ or $18.9 \%$ reduction from the base year. This can be attributed to EMCS and other energy conservation measures. There was a square footage increase from the baseline year of $2305 \mathrm{~K}$ square feet in 2000 to $2727 \mathrm{~K}$ square feet in 2002, an increase of $15.5 \%$. Using a straight ratio for energy demand, this means the example facility would have normally had an increase in energy use of $15.5 \%$ over this period if it had not been for the energy reduction measures taken.

Putting a dollar value to this reduction is much more difficult because of price fluctuations from month to month. Using cost averaging for the base year and the current total cost per mBtu for the current year to calculate real savings, the numbers are as indicated below. Savings are calculated from reduction $\mathrm{x}$ dollars per Btu for that year $\mathrm{Z}$ square footage (reduced x \$/Btu/Zsq ft) .

To date, this amounts to $\$ 1.3$ million dollars in accumulated savings from base year of avoided cost in energy only. No credit has been taken for labor savings. The system has only been in operation for 2 full years, thus 2001 and 2002 are the only years that show savings. 


\section{Commissioning EMCS}

Without complete and through commissioning of the EMCS, there is no guarantee that the functions and capabilities will work as intended. Most often even the new systems are not adequately commissioned leading to incomplete and inefficient operation of the EMCS. Sufficient resources should be allocated to commission the EMCS after it is installed to ensure that it works as intended.

Often during installation, key pieces of hardware are either missing, not installed, or defective. Other common problems include 1 . The installation of sensors and thermostats in the wrong locations, and/or 2. Improper coding of the sequence of controls for equipment. These and other problems are identified and corrected by a good control system commissioning process.

Recommendation: We recommend that control system specifications require a thorough commissioning of EMCS as part of EMCS procurement and that technicians on-site with end ownership verify same. 


\section{Operator Skills and Training}

One of the keys to enhancing building operations and maintenance and thereby achieving the efficiency goals of buildings/facilities are well trained building operators and managers. Lack of properly trained operators and high turnover of building operators contributes significantly to inefficient building operation and maintenance. Performance problems result from errors in installation and operation of complex building heating/cooling systems and particularly their controls. Furthermore, HVAC systems are becoming increasingly more sophisticated to obtain ever-higher levels of energy efficiency, adding to the complexity and subtlety of problems that reduce the net efficiency acquired. Without the right skill sets and proper training, building operators and managers may not be able to manage the facility optimally even with the most advanced EMCS. To operate buildings efficiently, in addition to a good controls infrastructure, the following is also necessary:

1. Increase the skill level of operators and maintenance personnel. This can be accomplished by providing the building operators with frequent proper training and incentives for performing well.

2. Provide adequate engineering supervision of technician work to ensure that adequate knowledge of fundamental processes is brought to bear on operation and maintenance actions.

3. Provide operators with system performance feedback. This includes not just reporting alarms through an EMCS, but also providing easy-to-use system diagnostic information to correct problems and to understand the cost impacts from improper operations.

4. Provide incentives for correcting problems. There is a general lack of motivation among operators to identify the root cause when a problem arises. More often operators try to correct the symptom of the problem by changing setpoints or controls rather than determining the root cause and correcting it.

5. Provide incentives for achieving efficiency goals. Most often when building operators work hard to achieve the efficiency goal set for the building, they are rewarded with a reduced energy budget corresponding to the new lower consumption level. This budget reduction may be interpreted by engineering and operations staff as a disincentive. Attention should be given, instead, to ensuring that operators are provided positive reinforcement for good performance.

6. Educate everyone who influences the planning, budget, design, and procurement of energy and related systems. 


\section{Control-System Maintenance}

Maintenance of the control system is critical to ensuring its performance after installation and commissioning. Commissioning ensures that the control system operates properly at only one point in time, the time immediately following the completion of commissioning. Many factors can cause degradation of the control system's performance afterward:

- Overrides of automatic control by operations staff, a frequent problem, generally driven by customer complaints

- Drift or failure of sensors

- Failure of actuators

- Corroded or failed wires and their connections

- Improper changes to control schedules (sometimes temporary changes become permanent changes by oversight)

- Degradation or failure of controlled devices.

These problems can only be prevented by good operation and maintenance practices, such as a reporting system for all overrides, periodic recommissioning of the control system, and maintenance of the control system and the physical components it actuates. As cited earlier in this document, automated fault detection and diagnostic tools, alarms, plots of trend data, and maintenance tracking systems can be used to help with identifying performance degradation, faults and the need for maintenance, but even these tools cannot ensure that the control system continues to operate properly unless the maintenance staff heeds the results and recommendations of these tools and takes action.

Recommendation: We recommend that the control system specifications include requirements for maintenance documents and a maintenance plan. Furthermore, the facility should also consider contracting for maintenance support if it will not be provided in-house. In procuring maintenance support, the organization should ensure that the maintenance contract requires maintenance of all systems in a manner that ensures continuous, or nearly continuous proper operation of all controls and controlled devices. This requires frequent, periodic inspection, testing, and evaluation of systems and equipment followed by the correction of any defects found. System inspection, testing and evaluation can be automated via continuous monitoring tools. 
